\title{
Relação entre o consumo de macronutrientes e a menarca em adolescentes
}

\author{
Relationship between consumption of \\ macronutrients and the menarche in adolescents
}

\author{
Ana Cristina Miguez Teixeira', Eduardo Borba Neves², \\ Evelise Fernandes Pietrovski ${ }^{3}$, Luis Paulo Gomes Mascarenhas ${ }^{4}$
}

\begin{abstract}
Resumo
Objetivo: Identificar a relação da ingestão de macronutrientes e a idade da menarca em jovens de Curitiba. Metodologia: Foram avaliadas 400 meninas divididas em dois grupos de acordo com a ingesta de gordura saturada da alimentação. Mensurouse a estatura e o peso corporal, calculou-se o IMC. A idade da menarca foi obtida por autorrelato. Para avaliar o consumo alimentar das jovens participantes, o questionário de frequência de consumo alimentar (QFCA) foi aplicado. Para análise dos dados foi utilizada a estatística descritiva, o teste $t$ de student e regressão linear stepwise, com nível de significância de 0,05. Resultados: Diferenças significativas não foram observadas para as variáveis de composição corporal, apenas para a idade da menarca sendo menor no grupo de meninas que ingeriam menos de 10\% de ácidos graxos saturados (G2). Foi observada uma maior ingestão pelo grupo das meninas que ingerem mais de 10\% de ácidos graxos saturados (G1) nas variáveis calorias consumidas, proteínas, gordura total, colesterol e AGSA. Na regressão linear para a idade da menarca, observou-se o consumo de ácido graxo saturado com valores $r=0,232$ e $r^{2}=0,05$. Conclusão: Dos macronutrientes avaliados, os ácidos graxos saturados apresentaram correlação com a idade da menarca.
\end{abstract}

Palavras-chave: menarca; nutrição do adolescente; ácidos graxos.

\begin{abstract}
Objective: Identify the relationship between macronutrient intake and age at menarche in young girls of Curitiba-PR. Methodology: A total of 400 girls divided into two groups according to the intake of saturated fat diet. Was measured height and weight, and after, calculated BMI. The age of menarche was obtained by self-report. To assess the nutritional intake of young participants, the questionnaire frequency of food consumption (FFQ) was applied. For data analysis we used descriptive statistics, Student's t test and stepwise linear regression with a significance level of 0.05 . Results: Significant differences were observed for body composition variables, only age at menarche was lower in the group of girls who consumed less than $10 \%$ of saturated fatty acids (G2). There was a higher intake of protein, total fat, cholesterol and AGSA by the group of girls who eat more than $10 \%$ of saturated fatty acids (G1). By linear regression analysis it was found that consumption of saturated fatty acids may influence age of menarche and $r=0.232$ and $r^{2}=0.05$. Conclusion: Of the nutrients studied, the saturated fatty acids presented correlated with age at menarche.
\end{abstract}

Keywords: menarche; adolescent nutrition; fatty acids; metabolic syndrome.

Trabalho realizado no Centro Universitário Campos de Andrade (UNIANDRADE) - Curitiba (PR), Brasil

1Professora Doutora do Curso de Nutrição do UNIANDRADE - Curitiba (PR), Brasil.

2Professor Doutor do Programa de Pós-graduação em Engenharia Biomédica da Universidade Tecnológica Federal do Paraná (UTFPR) - Curitiba (PR), Brasil.

3Professora Doutora do Curso de Farmácia do UNIANDRADE - Curitiba (PR), Brasil.

${ }^{4}$ Professor Doutor do Programa de Pós-graduação em Desenvolvimento Regional da Universidade do Contestado (UnC) - Canoinhas (SC), Brasil. Contato para correspondência: Eduardo Borba Neves - Rua Marquês do Paraná, 418, apto. 104 - CEP: 80620-210 - Curitiba (PR), Brasil - E-mail: borbaneves@hotmail.com

Fonte de financiamento: nenhuma.

Conflito de interesse: nada a declarar. 


\section{INTRODUÇÃO}

Pesquisas realizadas demonstram que a menarca está se manifestando cada vez mais precocemente nas jovens do mundo inteiro ${ }^{1}$. No Brasil, esta situação apresenta-se prevalente, o que preocupa profissionais de saúde. Aspectos nutricionais e psicológicos afetam as meninas que são influenciadas em sua maturidade sexual através de estímulos visuais e estilo de vida, em especial a alimentação.

Petridou et al. ${ }^{2}$ demonstraram existir relação entre a ingestão de gordura, macronutriente, variáveis antropométricas e idade de menarca materna, como preditores da idade de início da menarca. Em estudo realizado no Canadá, com meninas entre seis e 14 anos, em fase de pré-menarca, Koo et al. ${ }^{3}$, observaram que a ingestão de uma quantidade maior de fibra dietética foi associada com a menarca mais tardia.

Em contrapartida, Maclure et $a .^{4}$, demonstraram que a menarca não está associada à ingestão de macronutrientes. Porém, os resultados desta pesquisa são consistentes em relação a fatores nutricionais e à idade da menarca, principalmente ao ganho de tecido adiposo.

Um grupo de 939 meninas de várias etnias, entre as idades de oito a 13 anos, foi estudado na Califórnia do Sul (EUA) durante quatro anos para avaliar fatores relacionados à menarca, considerada um fator de risco para câncer de mama. De todas as relações avaliadas, apenas a ingestão energética foi relacionada com a idade da menarca. A altura associada à baixa ingestão energética foi preditor de atraso na menarca ${ }^{5}$.

Em uma meta-análise realizada por Perry et al. ${ }^{6}$, comprovouse a existência de genes que influenciam maturidade sexual nas jovens e, consequentemente, influenciam a idade da menarca. Alves et al. ${ }^{7}$ chamam a atenção para os produtos, interferentes endócrinos, que, eventualmente, podem estar contaminados como carnes e produtos, como a soja, que é, comprovadamente, repositora hormonal. Eles sugerem que a exposição ambiental pode estar associada à atividade estrogênica e, consequentemente, a distúrbios puerperais em crianças.

A falta de concordância científica quanto aos fatores que estimulam a puberdade cada vez mais precoce aponta a necessidade da realização de mais estudos que contribuam para que se alcance um consenso sobre o assunto. Neste sentido, o objetivo deste estudo foi identificar a relação da ingestão de macronutrientes e a idade da menarca em jovens escolares da rede pública da cidade de Curitiba (PR).

\section{MÉTODOS}

Este estudo teve abordagem quantitativa de caráter analítico e transversal e foi aprovado pelo Comitê de Ética em Pesquisa em Seres Humanos da Universidade Federal do Paraná sob o registro 1466.131/2007-06 e CAAE: 0137.0.208.00-07, observando a resolução 196/96 do Conselho Nacional de Saúde.

A população deste estudo pode ser definida como as meninas matriculadas nas escolas da rede de ensino públicas da cidade de Curitiba com idade entre 10 e 18 anos.

Para determinação da amostra, foi realizada uma análise probabilística de acordo com o sexo, e em seguida, o tamanho amostral foi avaliado de acordo com os seguintes critérios: a) número total meninas; b) intervalo de confiança de $95 \%$; c) erro amostral de $5 \%$ e prevalência de $50 \%$, uma vez que a prevalência dos fatores de risco nesta população é desconhecida.

Prevendo-se possíveis desistências, perda de dados durante o período de avaliação e a possibilidade de evasão escolar de $0,07 \%$ no estado do Paraná, optou-se por acrescentar uma quantidade de $10 \%$ de avaliados, garantindo a representatividade da amostra. Foi calculado número mínimo de indivíduos para representatividade da escola, contudo, todas as alunas tiveram oportunidade igual de participar do estudo.

A amostra casual foi composta por 972 adolescentes de ambos os gêneros, com idades entre 10 e 18 anos, matriculadas nas escolas da rede de ensino públicas da cidade de Curitiba, PR. Destas, 552 meninas iniciaram o estudo, mas apenas 400 realizaram todas as fases do mesmo.

A medida da estatura foi obtida avaliando a maior distância do chão até o vértex da cabeça, por meio de um estadiômetro vertical portátil (WCS ${ }^{\circledast}$, Brasil), escalonado em $0,1 \mathrm{~cm}$. O avaliado estava em posição anatômica sobre a base do estadiômetro, manteve os calcanhares unidos e tocando a borda do equipamento. A haste do aparelho foi colocada no ponto mais alto da cabeça, de maneira a formar um ângulo reto com a borda vertical do aparelho, e cabeça centralizada e posicionada anatomicamente na posição de Frankfort. As jovens foram orientadas a ficar em apneia inspiratória no momento da avaliação ${ }^{8}$.

A massa corporal das jovens foi aferida em uma balança digital portátil da marca Filizola ${ }^{\circledR}$ (Filizola, Brasil), com resolução de 100 gramas. As adolescentes foram avaliadas em roupas leves e descalças, ficando em pé sobre o centro da plataforma do equipamento e de costas para a escala, em posição anatômica, de maneira a distribuir igualmente a massa corporal em ambos os pés 8 .

O grau de maturação sexual foi avaliado na forma de autoavaliação considerando os critérios de Tanner'. A autoavaliação foi realizada com a apresentação de desenhos e relatada individualmente pela avaliada. No desenho havia um texto explicativo. $\mathrm{O}$ exame realizado em forma de autoavaliação da pilosidade pubiana é considerado um processo simples que pode ser realizado pela própria criança ou adolescente ${ }^{10}$. Em estudo realizado com adolescentes brasileiros, uma 
concordância de $89 \%$ foi encontrada entre a autoavaliação da maturação sexual e a avaliação médica ${ }^{11}$.

Para avaliar o consumo alimentar das jovens participantes, foi utilizado o questionário de frequência de consumo alimentar (QFCA). Além de apresentar boa reprodutibilidade, tem validade aceitável, é de prático manuseio, informativo, de baixo custo e de fácil aplicação ${ }^{12}$. O QFCA foi desenhado para fornecer informações quantitativas e qualitativas sobre o padrão alimentar e a ingestão de alimentos ou nutrientes específicos ${ }^{13}$. O QFCA foi desenvolvido e validado para a população brasileira pediátrica por Sichieri e Everhart e por Fonseca et al. O questionário foi aplicado em forma de autoavaliação ${ }^{14,15}$.

O QFCA contém uma lista com 82 itens alimentares, contendo o registro de frequência de consumo alimentar em intervalos de tempo (dia, semana e mês) e informações sobre a quantidade de porção dos alimentos. $\mathrm{Na}$ análise do questionário levou-se em consideração, para o cálculo dos nutrientes, o banco de dados de composição de alimentos do Programa de Apoio à Nutrição, desenvolvido pela Escola Paulista de Medicina ${ }^{16}$. Em posse destes valores, foi construída uma planilha para transformar o consumo em frequências diárias e as quantidades das porções multiplicadas pelos valores de referência. Finalmente, as quantidades diárias foram transformadas em nutrientes e quantidade de energia (calorias). Com estes dados foi possível calcular a quantidade média de quilocalorias ingeridas por dia (kcal/dia), gordura total (g), gorduras saturadas (g) e colesterol (mg). Foram utilizados como pontos de corte para exclusão, os valores de consumo energéticos superiores a $600 \mathrm{kcal}$ ou inferior a $500 \mathrm{kcal}^{16}$.

A adequação dos sujeitos em relação aos indicadores dietéticos foi realizada mediante as recomendações da Academia Americana de Pediatria17, a qual considera como consumo alimentar adequado $\leq 10 \%$ de gordura saturada e $\leq 300 \mathrm{mg} /$ dia de colesterol. A partir destas informações, dividiram-se as meninas em dois grupos, sendo o grupo 01 (G1) adolescentes que consumiam mais de 10\% de gordura saturada em sua dieta e o grupos $02(\mathrm{G} 2)$ aquelas que consumiam menos de $10 \%$ de gordura saturada ${ }^{17}$.

Para a caracterização da amostra foi utilizada a estatística descritiva expressa em valores médios e desvio padrão. A comparação das variáveis dependentes entre os grupos de meninas que ingerem mais de $10 \%$ de ácidos graxos saturados (G1) e que ingerem menos de $10 \%$ de ácidos graxos saturados (G2) em sua dieta foi realizada através do teste $t$ Student para amostras independentes, além da análise de regressão linear com um nível de significância estipulado em $\mathrm{p}<0,05$. Todos estes procedimentos foram realizados no software estatístico SPSS 13.0 (Chicago, IL).

\section{RESULTADOS}

Os dados antropométricos de caracterização da amostra estão apresentados na Tabela 1, separados em grupos de meninas que ingerem mais de $10 \%$ de ácidos graxos saturados (G1) e que ingerem menos de $10 \%$ de ácidos graxos saturados (G2) em sua dieta, não sendo encontradas diferenças significativas com exceção para a idade da menarca, que foi menor nas adolescentes do grupo G2. Pela análise de regressão linear se observa que o consumo de ácidos graxos saturados podem influenciar a idade da menarca em $r=0,232$ e $r^{2}=0,05$.

Os resultados do questionário alimentar demonstraram um maior consumo de calorias, proteínas, gordura total, ácidos graxos saturados e colesterol pela adolescente do grupo G1 (Tabela 2).

\section{DISCUSSÃO}

As variáveis antropométricas não apresentaram diferenças significativas entre os grupos demonstrando uma homogeneidade da amostra. Segundo a FAO/WHO, tanto o peso quanto a altura encontrados no presente estudo podem ser classificados como adequados. Quanto aos valores médios encontrados ambos os grupos se localizam dentro da normalidade para o estado nutricional de acordo com as variáveis de CC e IMC ${ }^{18}$.

Tabela 1. Caracterização das adolescentes de Curitiba - PR, 2009

\begin{tabular}{lcccc} 
& G1 $(\mathbf{n}=\mathbf{3 0 1})$ & G2 $(\mathbf{n}=99)$ & Valor t & Valor $\mathbf{p}$ \\
Idade (anos) & $14,06 \pm 2,13$ & $14,55 \pm 2,94$ & $-0,90$ & 0,37 \\
Estatura $(\mathrm{cm})$ & $156 \pm 0,8$ & $154 \pm 0,8$ & 1,44 & 0,15 \\
Peso corporal $(\mathrm{kg})$ & $50,55 \pm 10,74$ & $52,02 \pm 14,73$ & $-1,06$ & 0,28 \\
Massa magra $(\mathrm{kg})$ & $34,67 \pm 6,19$ & $34,16 \pm 8,73$ & 0,55 & 0,58 \\
Massa gorda $(\mathrm{kg})$ & $15,88 \pm 7,5$ & $16,80 \pm 11,34$ & 0,65 & 0,51 \\
CC $(\mathrm{cm})$ & $66,57 \pm 7,76$ & $68,55 \pm 11,28$ & $-1,58$ & 0,11 \\
IMC $\left(\mathrm{kg} / \mathrm{m}^{2}\right)$ & $20,53 \pm 3,49$ & $21,42 \pm 4,85$ & $-1,65$ & 0,10 \\
Menarca (anos) & $11,97 \pm 1,16$ & $11,60 \pm 1,26$ & 2,07 & $0,03^{*}$ \\
\hline
\end{tabular}

*diferença significativa entre os grupos

Tabela 2. Resultados do consumo de nutrientes nas adolescentes de Curitiba - PR, 2009

G1 $(\mathrm{n}=301) \quad \mathrm{G} 2(\mathrm{n}=99) \quad$ Valor $\mathrm{t}$ Valor $\mathrm{p}$

Calorias (kcal) $\quad 2755,40 \pm 806,58 \quad 2399,35 \pm 887,93 \quad 3,69 \quad 0,001^{\star}$

Proteínas (g) $\quad 101,88 \pm 32,66 \quad 81,93 \pm 31,08 \quad 5,30 \quad 0,001^{*}$

Carboidratos (g) $\quad 375,30 \pm 118,62 \quad 392,02 \pm 141,16 \quad-1,15 \quad 0,42$

Gordura total (g) $\quad 98,04 \pm 37,81 \quad 60,84 \pm 32,24 \quad 8,53 \quad 0,001^{\star}$

Colesterol (mg) $\quad 365,65 \pm 252,41 \quad 201,93 \pm 206,63 \quad 5,83 \quad 0,001^{\star}$

AGSA (g) $\quad 41,13 \pm 13,63 \quad 25,01 \pm 17,13 \quad 6,32 \quad 0,001^{*}$

AGPO (g) $\quad 13,60 \pm 7,58 \quad 19,97 \pm 9,32 \quad-1,01 \quad 0,31$

*diferença significativa entre os grupos 
Em relação ao valor calórico e considerando-se a média de peso entre os dois grupos estudados, percebe-se que os resultados demonstram uma ingestão energética maior do que a prevista para a idade e peso apenas no G1. Para a RDA (Recommended Dietary Allowances) ${ }^{19}$, o valor calórico médio recomendado para adolescentes é de 2052,3 kcal/dia, considerando-se um fator de atividade leve. A ingestão calórica recomendada pela FAO (Food and Agriculture Organization) ${ }^{20}$ ficaria ao redor de $2.449 \mathrm{kcal} / \mathrm{dia}$ ou $47,0 \mathrm{kcal} / \mathrm{kg} / \mathrm{dia}$, sendo que a média de ganho de peso de $3,4 \mathrm{~kg} /$ ano seria esperada para este tipo de ingesta.

Quanto à ingestão de proteína ambos os grupos estão de acordo com a recomendação da $\mathrm{OMS}^{21}$ que é de 10-15\%/dia, verificou-se que o G1 ingeriu 14,79\%/dia e o G2 13,66\%/dia. Contudo, cuidados devem ser dados à ingestão excessiva de proteínas, pois pode levar à sobrecarga das funções renais, aumentando o ritmo de filtração glomerular ${ }^{22}$. Weijs et $\mathrm{al}^{23}$ demonstraram que a ingestão excessiva de proteína animal associado a açúcares simples, durante a infância, pode aumentar as chances de desenvolvimento de sobrepeso ainda na pré-adolescência.

Para o consumo de carboidratos, a recomendação da $\mathrm{OMS}^{21}$ é de $55-75 \% /$ dia, sendo que menos de $10 \%$ devem ser provenientes de açúcares livres. O G1 ingeriu 54,48\%/dia e o G2 ingeriu 65,35\%/dia, em relação ao valor calórico. O grupo G2 consumiu $10 \%$ a mais deste macronutriente sendo que uma atenção especial deve ser dada a ingestão excessiva dos refinados pela sua relação direta com o risco de desenvolvimento de doenças como o Diabetes Mellitus e Síndrome Metabólica ${ }^{24}$.

A ingestão de gorduras totais recomendada pela $\mathrm{OMS}^{21}$ é de $15-30 \% / d i a$, sendo que ambos os grupos ficaram dentro do recomendado com o G1 ingerindo 32,02\%/dia e o G2 ingeriu $22,82 \% /$ dia. Observa-se a ingestão foi mais elevada no G1 em cerca de 2,02\% acima da recomendação.

O G1 ingeriu $20 \%$ a mais do recomendado de colesterol total e o G2 apresentou-se dentro da faixa de normalidade. A recomendação da $\mathrm{OMS}^{21}$ é de menos de $300 \mathrm{mg} /$ dia.

Com referência ao consumo de ácidos graxos saturados, o recomendado para ingestão diária é de menos de $10 \%$ do valor calórico ingerido no dia. O G1 teve uma ingestão de 41,13 g, o que representa $13,43 \%$ em relação ao valor calórico ingerido e o G2 apresentou uma ingestão de 9,53\%.

Em relação ao consumo de ácidos graxos poli-insaturados, a recomendação é de 6-10\% do valor calórico ingerido no dia, sendo $5-8 \%$ de ômega-6 e 1-2\% de ômega-3. O G1 apresentou consumo de $4,44 \%$ e o G2 de $0,83 \%{ }^{25}$.

$\mathrm{O}$ resultado relevante cientificamente deste estudo foi a associação positiva entre a idade da menarca e consumo de ácidos graxos saturados pelas adolescentes, colaborando com Maclure et al. ${ }^{26}$, que observaram resultados semelhantes, aonde uma menor ingesta de ácidos graxos saturados esteve presente nas meninas com menor idade de menarca.

Uma possível explicação para o fato de uma baixa concentração de ácidos graxos saturados influenciarem na idade da menarca pode estar na relação inversa encontrada com a concentração de adiponectina ${ }^{27}$.

Donoso et al. ${ }^{28}$ pesquisaram os níveis de adiponectina $\mathrm{e}$ leptina em bailarinas e observaram que a elevação na leptina e uma redução da adiponectina são agentes do atraso na menarca das bailarinas. Apesar de não ter sido avaliado no presente estudo e representar uma limitação do estudo, a relação entre ingesta de ácidos graxos e alteração hormonal precisam ser melhores elucidadas.

É importante mencionar a associação da idade da menarca com a distribuição de gordura corporal. Destaca-se que a distribuição da gordura corporal tanto na região da cintura quanto no tríceps aumentam o risco de menarca em 7 e 9\%, sendo mais importante do que a gordura total e a maturidade esquelética ${ }^{29}$. Entretanto, no presente estudo a CC não se manifestou significativa na análise de regressão possivelmente porque os grupos não se diferiram. Contudo, não se pode descartar a possível influência da adiposidade da região triceptal, a qual não foi avaliada.

Apesar de não se manifestar significativamente sobre a idade da menarca, a ingestão de ácidos graxos poli-insaturados deve ser estimulada para uma maior qualidade de vida, pois, Kariyazono e Nakamura ${ }^{30}$ demonstram que ácidos graxos poli-insaturados modulam processos inflamatórios e contribuem para a fisiopatologia nos estados leves de inflamação.

\section{CONCLUSÃO}

Sabe-se que a alimentação em nível mundial e no Brasil, nem sempre está dentro do esperado para garantir uma nutrição adequada e qualidade de vida dos adolescentes. A situação destes estados de subnutrição e de superalimentação demonstra a demanda de educação nutricional.

Identificou-se, no presente estudo, que a baixa ingestão de ácido graxo saturado apresenta relação com o início da menarca. Quando subdividida a amostra entre baixa e alta ingestão de ácido graxo saturado observou-se, também, uma diferença significativa na ingestão dos macronutrientes entre os grupos. Futuras pesquisas relacionando a ingestão de macronutrientes, marcadores inflamatórios e menarca precisam ser realizadas para melhor elucidar esta relação. 


\section{REFERÊNCIAS}

1. Cabanes A, Ascunce N, Vidal E, Ederra M, Barcos A, Erdozain N et al. Decline in age at menarche among Spanish women born from 1925 to 1962. BMC Public Health. 2009;9:449.

2. Petridou E, Syrigou T, Toupadaki N, Zavitsanosw X, Willelt A, Trichopoulos D. Determinants of age at menarche as early life predictors of breast cancer risk. Int J Cancer. 1996;68:193-198.

3. Koo M M, Rohan T E, Jain M, McLaughlind J R, Corey P N. A cohort study of dietary fibre intake and menarche. Public Health Nutr. 2001;5:353-60.

4. Maclure M, Travis LB, Willett W, MacMahon B. A prospective cohort study of nutrient intake and age at menarche. Am J Clin Nutr. 1991;54:649-56.

5. Koprowski C, Ross RK, Mack WJ, Henderson BE, Bernstein L. Diet, body size and menarche in a multiethnic cohort. Br J Cancer. 1999;79:1907-11.

6. Perry JRB, Stolk L, Franceschini $\mathrm{N}$ et al. Meta-analysis of genome-wide association data identifies two loci influencing age at menarche. Nat Genet. 2009;41:648-50.

7. Alves C, Flores LC, Cerqueira TS, Toralles MB. Exposição ambiental a interferentes endócrinos com atividade estrogênica e sua associação com distúrbios puberais em crianças. Cad Saúde Públ. 2007;23:1005-14.

8. Docherty D. Measurement in pediatric exercise science. Hum Kinet; 1996.

9. Tanner JM. Growth and adolescence. Blackwell Scient Public. Oxford; 1962.

10. Martin RHC, Uezu R, Parra SA, Arena SS, Bojikian LP, Bohme MTS. Auto-avaliação da maturação sexual masculina por meio da utilização de desenhos e fotos. Rev Paul Ed Fís. 2001;15:212-22.

11. Matsudo SM, Matsudo VK. Self-assessment and physician assessment of sexual maturation in Brazilian boys and girls: concordance and reproducibility. Am J Human Biol. 1994;6:451-55.

12. Cavalcante AAM, Priore SE, Franceschini SCC. Estudos de consumo alimentar: aspectos metodológicos gerais e seu emprego na avaliação de crianças e adolescentes. Rev Bras Saúde Mat Infant. 2004;4:229-40.

13. Fisberg RG, Slater B, Marchioni DML, Martini LA. Inquéritos alimentares: métodos e bases científicas. São Paulo. Manole: 2005.

14. Sichieiri R, Everhart JE. Validity of brazilian food frequency questionnaire against dietary recalls and estimated energy intake. Nutr Res. 1998;18:1649-59.

15. Fonseca VM, Sichieri R, Veiga V. Fatores associados à obesidade em adolescentes. Rev Saúde Públ. 1998;32:541-49.

16. Andrade RG, Pereira RA, Sichieri R. Consumo alimentar de adolescentes com e sem sobrepeso do município do Rio de Janeiro. Cad Saúde Públ. 2003;19:1485-95.
17. American Academy of Pediatrics Committee of Nutrition. Statement on cholesterol. Pediatrics. 1992;90:469-73.

18. Kuczmarski RJ, Ogden CL, Grummer-Strawn LM et al. CDC growth charts: United States. Advance data from vital and health statistics; n. 314 . Hyattsville, Maryland: National Center for Health Statistics, 2000.

19. National Research Council. Recommended Dietary Allowances, National Academy Press, 1989.

20. Food and Agriculture Organization of the United Nations. Human energy requirements: children and adolescents. Rome, 2004. Report of a Joint FAO/WHO/UNU Expert Consultation Food and Nutrition Technical report series 1 .

21. OMS, Serie de Informes Técnicos/916. Dieta, nutrición y prevención de enfermedades crônicas. Informe de uma Consulta Mixta de Expertos OMS/FAO. Organización Mundial de la Salud. Ginebra, 2003.

22. Higashiyama A, Watanabe M, Kokubo Y, Ono Y, Okayama A, Okamura T. Relationships between protein intake and renal function in a japanese general population: NIPPON DATA90. J Epidemiol. 2010;20.

23. Weijs PJM, Kool LM, van Baar NM, van der Zee SC. High beverage sugar as well as high animal protein intake at infancy may increase overweight risk at 8 years: a prospective longitudinal pilot study. Nutr J. 2011;10-95.

24. Oliveira EP, Souza MLA, Lima MDA. Prevalência de síndrome metabólica em uma área rural do semi árido baiano. Arq Bras Endocrinol Metabol. 2006;50:456-65.

25. FAO/WHO. Interim summary of conclusions and dietary recommendations on total fat \& fatty acids. Expert Consultation on Fats and Fatty Acids in Human Nutrition. 2008;10-14, WHO HQ, Geneva.

26. Maclure M, Travis LB, Willett W, MacMahon B. A prospective cohort study of nutrient intake and age at menarche. Am J Clin Nutr. 1991;54:649-56

27. Gallo S, Egeland G, Meltzer S, Legault L, Kubow S. Plasma fatty acids and desaturase activity are associated with circulating adiponectin in healthy adolescent girls. J Clin Endocrinol Metabol. 2010;95:2410-17.

28. Donoso MA, Munoz-Calvo MT, Barrios V, Garrido G, Hawkins F, Argente J. Increased circulating adiponectin levels and decreased leptin/ soluble leptin receptor ratio throughout puberty in female ballet dancers: association with body composition and the delay in puberty. Eur J Endocrinol. 2010;162:905-11.

29. Lassek WD, Gaulin SJC. Brief Communication: menarche is related to fat distribution. Am J Phys Anthropol. 2007;133:1147-51.

30. Kariyazono H, Nakamura K. Pleiotropic effects of dietary fatty acids and fatty acid involvement in chronic mild inflammation - related diseases. Kariyazono H, Nakamura K. J Health Sci. 2010;56:473-87.

Recebido em: 22/01/2013 Aprovado em: 15/05/2013 